\title{
INDUCTIVE AND PROJECTIVE LIMITS OF NORMED SPACES
}

\author{
by JOHN S. PYM
}

(Received 15 May, 1967)

Let $\left\{U_{i}, u_{i j}\right\}$ be an inductive system of normed linear spaces $U_{i}$ and continuous linear maps $u_{i j}: U_{j} \rightarrow U_{i}$. (We write $j \prec i$ if $u_{i j}: U_{j} \rightarrow U_{i}$.) An inductive limit of the system with respect to a class $(\mathfrak{U}, \mathfrak{m})$ of spaces $A$ in $\mathfrak{U}$ and maps $f$ in $\mathfrak{m}$ is a space $U_{\mathfrak{u}}$ in $\mathfrak{U}$ and a system $u_{i}: U_{i} \rightarrow U_{\mathfrak{u}}$ of maps in $\mathfrak{M}$ such that (i) $u_{i} \circ u_{i j}=u_{j}$ whenever $j \prec i$, and that (ii) if $A$ is any space in $\mathfrak{U}$ and $f_{i}: U_{i} \rightarrow A$ is any system of maps in $\mathfrak{M}$ for which $f_{i} \circ u_{i j}=f_{j}(j \prec i)$, then there is a unique map $f: U_{\mathfrak{u}} \rightarrow A$ in $\mathfrak{m}$ such that $f_{i}=f_{\circ} u_{i}$ for each $i$. If $\mathfrak{U}$ is the class of all vector spaces and $\mathfrak{M}$ is the class of linear maps, we obtain the algebraic inductive limit, which we denote simply by $U$. The usual choice is to take $\mathfrak{U}$ to be the class of locally convex spaces and $\mathfrak{m}$ the class of continuous linear maps; the inductive limit $U_{L}$ then always exists $[1, \S 16 \mathrm{C}]$. If $\mathfrak{M}$ is again the continuous linear mappings but $\mathfrak{U}$ contains only normed spaces, the corresponding inductive limit $U_{N}$ may not always exist. However, if in addition we require that $\mathfrak{m}$ contains just contractions (norm-decreasing linear mappings), then an inductive limit $U_{C}$ will exist if every $u_{i j}$ is a contraction [2]. We shall give a condition under which these limits coincide (as far as possible), and consider the corresponding condition for projective limits.

THEOREM 1. If $U_{N}$ exists, it is isomorphic (as a locally convex space) with $U_{L}$.

Proof. As $U_{N}$ is a locally convex space, there is a unique map $f: U_{L} \rightarrow U_{N}$ such that the composite maps $U_{i} \rightarrow U_{L} \stackrel{f}{\rightarrow} U_{N}$ are the canonical maps into $U_{N}$. We find a continuous linear inverse for $f$. Let $p$ be any continuous seminorm on $U_{L}$, and let $U_{p}=U_{L} / p^{-1}(0)$ be the normed quotient space. The continuous maps $U_{i} \rightarrow U_{L} \rightarrow U_{p}$ provide a continuous map $g_{p}: U_{N} \rightarrow U_{p}$, and the maps $g_{p}$ yield a continuous linear map $g$ of $U_{N}$ into the projective limit of the spaces $U_{p}$, viz. $U_{L}$ itself $[1, \S 16 \mathrm{D}(h)]$. It is easy to see that the maps $U_{i} \rightarrow U_{N} \rightarrow U_{L}$ are the canonical maps into $U_{L}$, and we deduce that both $f \circ g$ and $g \circ f$ are identity maps.

We shall say that $\left\{U_{i}, u_{i j}\right\}$ is countably directed (resp. directed) if, for any countable (resp. finite) set $\left\{i_{1}, i_{2}, \ldots\right\}$, there is a $j$ such that $i_{n} \prec j$ for every $n$. If the system is directed and each $u_{i j}$ is an injection, then the canonical maps $u_{i}: U_{i} \rightarrow U$ are injections. It is shown in [2] that if, in addition, $u_{i j}$ is a contraction for each $i, j$, then $\|x\|=\inf _{i}\left\|u_{i}^{-1}(x)\right\|_{i}$ (where $\|\cdot\|_{i}$ denotes the norm in $U_{i}$ ) defines a seminorm in $U$, and $U_{C}$ is the quotient of $U$ by the subspace $\{x:\|x\|=0\}$.

TheOREM 2. Let $\left\{U_{i}, u_{i j}\right\}$ be countably directed, and let each $u_{i j}$ be a contraction and an injection. Then, for each $x$, there is an $i$ such that $\|x\|=\left\|u_{i}^{-1}(x)\right\|_{i}$. Further, $\|\cdot\|$ is a norm on $U$, and $U_{N}$ and $U_{c}$ both exist and are isomorphic to $(U,\|\cdot\|)$. If each $U_{i}$ is a Banach space, so also is $(U,\|\cdot\|)$.

Proof. For $x \in U$, take $\left(i_{n}\right)$ such that $\left\|u_{i_{n}}^{-1}(x)\right\|_{i_{n}} \rightarrow\|x\|$. For any $j$ with $i_{n} \prec j$ for all $n$, we have $\|x\| \leqq\left\|u_{j}^{-1}(x)\right\|_{j}=\left\|u_{j i_{n}}\left(u_{i_{n}}^{-1}(x)\right)\right\|_{j} \leqq\left\|u_{i_{n}}^{-1}(x)\right\|_{i_{n}} \rightarrow\|x\|$; whence $\left\|u_{j}^{-1}(x)\right\|_{j}=\|x\|$. We can say more: if $\left\{x_{n}\right\}$ is any countable subset of $U$, there is a $j$ such that $\left\|u_{j}^{-1}\left(x_{n}\right)\right\|=\left\|x_{n}\right\|$ for each $n$ (for let $j_{n}$ satisfy $\left\|u_{j_{n}}^{-1}\left(x_{n}\right)\right\|_{j_{n}}=\left\|x_{n}\right\|$, and take $j$ with $j_{n} \prec j$ for all $n$ ). Therefore, if 
$\|x\|=0$, there is a $j$ such that $\left\|u_{j}^{-1}(x)\right\|_{j}=0$; whence $u_{j}^{-1}(x)=0$, and $x=0$. This and other elementary arguments show that $\|\cdot\|$ is a norm on $U$. Let $f_{i}: U_{i} \rightarrow A$ be any system of continuous linear maps into a normed space $A$ for which $f_{i} \circ u_{i j}=f_{j}$ whenever $j \prec i$, and let $f: U \rightarrow A$ be the canonical linear map for which $f_{\circ} u_{i}=f_{i}$ (all $i$ ). Put

$$
K=\sup \{\|f(x)\|:\|x\| \leqq 1\},
$$

and choose $\left(x_{n}\right)$ with $\left\|x_{n}\right\| \leqq 1$ such that $\left\|f\left(x_{n}\right)\right\| \rightarrow K$. We can find a $j$ such that $\left\|u_{j}^{-1}\left(x_{n}\right)\right\|_{j} \leqq 1$ for every $n$, and $\left\|f_{j}\left(u_{j}^{-1}\left(x_{n}\right)\right)\right\|=\left\|f\left(x_{n}\right)\right\| \rightarrow K$; therefore $K \leqq\left\|f_{j}\right\|$. We conclude that $f$ is continuous, and that if each $f_{j}$ is a contraction, so is $f$; thus $(U,\|\cdot\|)$ coincides with $U_{N}$ and $U_{C}$. Finally, if $\left(x_{n}\right)$ is a Cauchy sequence in $(U,\|\cdot\|)$, we can find a $j$ such that

$$
\left\|u_{j}^{-1}\left(x_{n}-x_{m}\right)\right\|_{j}=\left\|x_{n}-x_{m}\right\| \rightarrow 0
$$

as $m, n \rightarrow \infty$. If $U_{j}$ is complete, there is a $y$ such that $u_{j}^{-1}\left(x_{n}\right) \rightarrow y$; whence

$$
x_{n}=u_{j}\left(u_{j}^{-1}\left(x_{n}\right)\right) \rightarrow y \text {. }
$$

The completeness of each $U_{j}$ therefore implies the completeness of $(U,\|\cdot\|)$.

The notation we use for projective limits is parallel to that for inductive limits. The following result is obtained by arguments similar to those of Theorem 2 .

THEOREM 3. Let $\left\{V_{i}, v_{i j}\right\}$ be a countably directed projective system of normed spaces with each $v_{i j}$ a contraction. For $x \in V$, the algebraic projective limit, write $\|x\|=\sup _{i}\left\|v_{i}(x)\right\|_{i}$. Then there is an $i$ such that $\|x\|=\left\|v_{i}(x)\right\|_{i}$. Further, $\|\cdot\|$ is a norm on $V$, and $V_{N}$ and $V_{C}$ both exist and are isomorphic with $(V,\|\cdot\|)$. If each $V_{i}$ is a Banach space, so also is $(V,\|\cdot\|)$.

We remark that if $\left\{U_{i}, u_{i j}\right\}$ satisfies the hypotheses of Theorem 2, if $V_{i}$ is the normed dual of $U_{i}$, and if $v_{i j}$ is the adjoint of $u_{i j}$, then $\left\{V_{i}, v_{i j}\right\}$ satisfies the hypotheses of Theorem 3. It is shown in Theorem 2 of [2] that $V_{C}$ is the normed dual of $U_{C}$.

Examples. (i) Let $X$ be locally compact, and let $M(X)$ be the usual space of bounded Radon measures. For $\mu \geqq 0, \mu \in M(X)$, write $L^{1}(\mu)=\{v \in M(X): v \ll \mu\}$. The system of spaces $L^{1}(\mu)$ with the inclusion maps satisfies the conditions of Theorem 2 (countably directed because, given a sequence $\left(\mu_{n}\right)$, we can find a sequence $\left(a_{n}\right)$ of real numbers, with $a_{n}>0$ for each $n$, and $\left.\sum a_{n} \mu_{n} \in M(X)\right)$. It is easy to see that $M(X)$ is the (normed) inductive limit of these subspaces.

The dual of each $L^{1}(\mu)$ may be identified with $L^{\infty}(\mu)$. The normed dual of $M(X)$ is thus the normed projective limit of the spaces $L^{\infty}(\mu)$.

(ii) Even in this special situation, $V_{N}$ may not be isomorphic with $V_{L}$ (cf. Theorem 1). Thus take $X$ to be uncountable and discrete. Let $m$ be the (unbounded) measure which assigns mass 1 to each point. Then $M(X)=L^{1}(m)$, and $M(X)^{*}=L^{\infty}(m)$ is the space $B(X)$ of all bounded functions on $X$. The canonical projection from $B(X)$ to $L^{\infty}(\mu)$ maps the bounded function $f$ on $X$ to its restriction to the (necessarily countable) support of $\mu$. The locally convex space projective limit of the spaces $L^{\infty}(\mu)$ therefore consists of $B(X)$ with a topology defined by neighbourhoods $N(S, \varepsilon)=\{f:|f(x)|<\varepsilon$ for all $x \in S\} \quad(\varepsilon>0, S$ a countable subset of $X$ ). But, of course, $M(X)^{*}$ is $B(X)$ with the uniform norm. 
(iii) The limit $U_{N}$ may be distinct from $U_{C}$. Thus let $B$ be any Banach space, and let $U_{n}(n=1,2, \ldots)$ have the same underlying space as $B$, but with norm defined by $\|x\|_{n}=\|x\| / n$; the maps $U_{n} \rightarrow U_{m}$ are the identities if $n<m$. Then $U_{N}$ may be taken to be $B$; but $U_{C}=\{0\}$.

\section{REFERENCES}

1. J. L. Kelley and I. Namioka, Linear Topological Spaces, (Princeton, 1963).

2. Z. Semadini and $\mathbf{H}$. Zidenberg, Inductive and inverse limits in the category of Banach spaces, Bull. Acad. Polon. Sci. Sér. Sci. Math. Astr. Phys. 13 (1965), 579-583.

THE UNIVERSITY

SHEFFIELD 